\title{
Combination of Laplace transform and residual power series techniques to solve autonomous $n$-dimensional fractional nonlinear systems
}

https://doi.org/10.1515/nleng-2021-0022

Received Apr 30, 2021; accepted Aug 3, 2021.

\begin{abstract}
In this work, a new iterative algorithm is presented to solve autonomous $n$-dimensional fractional nonlinear systems analytically. The suggested scheme is combination of two methods; the Laplace transform and the residual power series. The methodology of this algorithm is presented in details. For the accuracy and effectiveness purposes, two numerical examples are discussed. Finally, the impact of the fractional order acting on these autonomous systems is investigated using graphs and tables.
\end{abstract}

Keywords: Caputo-fractional autonomous nonlinear systems, Laplace transform, residual power series method

\section{Introduction}

Extending the ordinary-partial differential equations into fractional differential equations has been attracted by many researchers since the fractional derivatives are more general, applicable and more efficient for real world phenomena, especially when the dynamics of a given mathematical model is affected by constraints inherent to the system [1]. Since it is difficult to find explicit solutions to these fractional problems, it is necessary to use, alternative methods, numerical and approximate techniques.

The most popular numerical techniques for solving fractional problems are the Collocation methods whose basic functions are of types: Haar functions, Legendre wavelets, Bernoulli polynomials, B-spline functions, Chebyshev polynomials, and others [2-6]. On the other

\footnotetext{
*Corresponding Author: Marwan Alquran, Department of Mathematics and Statistics, Jordan University of Science \& Technology, Irbid 22110, Jordan, E-mail: marwan04@just.edu.jo

Maysa Alsukhour, Mohammed Ali, Imad Jaradat, Department of Mathematics and Statistics, Jordan University of Science \& Technology, Irbid 22110, Jordan, E-mail: mmalsukhour19@sci.just.edu.jo, myali@just.edu.jo, iajaradat@just.edu.jo
}

side, many effective analytical schemes were developed to treat nonlinear problems involving fractional derivatives, such schemes are: generalized power series [13, 16, 22, 23], residual power series method [17, 26-29], differential transform method [19, 21, 24, 25], homotopy perturbation method $[14,15,18,20]$ and others.

In this work, we are interested in introducing a new analytical scheme to solve nonlinear fractional autonomous dynamical systems. Dynamical systems describe the prediction of future states follow from the current state. Different numerical and analytical algorithms were used to solve fractional dynamical systems, such as, Homotopy analysis method, the variational iteration method, Ritz method and the explicit one-step method [7-10]. In this context, we present a new algorithm constructed by combining the Laplace transform method and the residual power series method (LRPS). This new technique has been recently proposed for the first time in [11] and used in [12].

The organization of the paper is the following: In Section 2 we present the steps of applying the LRPS method to solve $n \times n$ autonomous fractional dynamical systems. In Section 3 we study two examples of order $2 \times 2$ and $3 \times 3$, and also provide graphical analysis. Finally, the conclusion is given in Section 4.

\section{Description of LRPS}

In this section, we present in details the steps of applying the LRPS scheme in solving the following autonomous fractional system:

$$
\left\{\begin{aligned}
D_{t}^{\alpha} v_{1}(t) & =h_{1}\left(t, v_{1}(t), v_{2}(t), \ldots, v_{n}(t)\right), \\
D_{t}^{\alpha} v_{2}(t) & =h_{2}\left(t, v_{1}(t), v_{2}(t), \ldots, v_{n}(t)\right), \\
& \cdot \\
& \cdot \\
& \cdot \\
D_{t}^{\alpha} v_{n}(t) & =h_{n}\left(t, v_{1}(t), v_{2}(t), \ldots, v_{n}(t)\right),
\end{aligned}\right.
$$


subject to the initial conditions

$$
v_{m}(0)=a_{m}, m=1,2,3, \ldots, n,
$$

where $0<\alpha \leq 1,0 \leq t \leq 1, h_{m}$ are suitable functions, and $D_{t}^{\alpha}$ is the Caputo-derivative.

Applying the Laplace transform to (1) we get

$$
V_{m}(s)=\frac{a_{m}}{s}+\frac{H_{m}(s)}{s^{\alpha}},
$$

where

$$
\begin{aligned}
V_{m}(s) & =\mathcal{L}\left[v_{m}(t)\right] \\
H_{m}(s) & =\mathcal{L}\left[h_{m}\left(t, v_{1}(t), v_{2}(t), \ldots, v_{n}(t)\right)\right] .
\end{aligned}
$$

We assume that $V_{m}(s), m=1,2,3, \ldots, n$ have fractional power series representation, i.e.,

$$
V_{m}(s)=\sum_{r=0}^{\infty} \frac{c_{m r}}{s^{r \alpha+1}}
$$

Next, we let $V_{m}^{k}(s)$ denote the $k$-th truncated series of $V_{m}(s)$, i.e.,

$$
V_{m}^{k}(s)=\sum_{r=0}^{k} \frac{c_{m r}}{s^{r \alpha+1}} .
$$

By condition (2), the 0-th LRPS approximate solution of $V_{m}(s)$ is $v_{m}(0)=\lim _{s \rightarrow \infty} s V_{m}(s)=c_{0 r}=a_{m}$. Thus,

$$
V_{m}^{k}(s)=\frac{a_{m}}{s}+\sum_{r=1}^{k} \frac{c_{m r}}{s^{r \alpha+1}} .
$$

Now, we define the Laplace residual function and the $k$-th Laplace residual function to (3), respectively, as:

$$
\begin{aligned}
& \operatorname{LRes}_{m}(s)=V_{m}(s)-\frac{a_{m}}{s}-\frac{H_{m}(s)}{s^{\alpha}}, \\
& \operatorname{LRes}_{m}^{k}(s)=V_{m}^{k}(s)-\frac{a_{m}}{s}-\frac{H_{m}(s)}{s^{\alpha}} .
\end{aligned}
$$

To determine the coefficients $c_{m r}, \quad m=1,2,3, \ldots, n$ and $r=1,2,3, \ldots, k$, we substitute (6) into (8), then multiply the resulting equation by $s^{k \alpha+1}$, and next we solve the following iterative equation

$$
\lim _{s \rightarrow \infty}\left(s^{k \alpha+1} \operatorname{LRes}_{m}^{k}(s)\right)=0,
$$

for the unknowns $c_{m r}: k=1,2,3, \ldots$. Finally, we apply the Laplace inverse to $V_{m}^{k}(s)$ to obtain the $k$-th approximate solution $v_{m}^{k}(t)$.

\section{Numerical Problems and concluding remarks}

In this section we present two examples of fractional nonlinear autonomous systems of order $2 \times 2$ and $3 \times 3$. The steps of implementing the LRPS will be clarified, and the effectiveness of the proposed method will be tested by providing a graphical analysis.

\subsection{Example 1}

Consider the following $2 \times 2$ nonlinear autonomous system $[7,8]$

$$
\left\{\begin{array}{l}
D^{\alpha} v_{1}(t)=\frac{v_{1}(t)}{2} \\
D^{\alpha} v_{2}(t)=v_{1}^{2}(t)+v_{2}(t)
\end{array}\right.
$$

subject to

$$
\begin{aligned}
& v_{1}(0)=1, \\
& v_{2}(0)=0 .
\end{aligned}
$$

Applying the Laplace transform to (10)-(11), we get

$$
\begin{aligned}
& V_{1}(s)=\frac{1}{s}+\frac{V_{1}(s)}{2 s^{\alpha}}, \\
& V_{2}(s)=\frac{1}{s^{\alpha}}\left(\mathcal{L}\left[\mathcal{L}^{-1}\left[V_{1}(s)\right]^{2}\right]+V_{2}(s)\right) .
\end{aligned}
$$

We assume that both $V_{1}(s)$ and $V_{2}(s)$ have fractional power series representation as

$$
\begin{aligned}
& V_{1}(s)=\sum_{n=0}^{\infty} \frac{a_{n}}{s^{n \alpha+1}}, \\
& V_{2}(s)=\sum_{n=0}^{\infty} \frac{b_{n}}{s^{n \alpha+1}},
\end{aligned}
$$

and we assume that the $k$-th truncated series of $V_{1}(s)$ and $V_{2}(s)$ are

$$
\begin{aligned}
& V_{1}^{k}(s)=\sum_{n=0}^{k} \frac{a_{n}}{s^{n \alpha+1}}=\frac{1}{s}+\sum_{n=1}^{k} \frac{a_{n}}{s^{n \alpha+1}}, \\
& V_{2}^{k}(s)=\sum_{n=0}^{k} \frac{b_{n}}{s^{n \alpha+1}}=\sum_{n=1}^{k} \frac{b_{n}}{s^{n \alpha+1}} .
\end{aligned}
$$

It is clear that the Laplace residual function for both $V_{1}^{k}(s)$ and $V_{2}^{k}(s)$ are

$$
\begin{aligned}
& \operatorname{LRes}_{1}(s)=V_{1}(s)-\frac{1}{s}-\frac{V_{1}(s)}{2 s^{\alpha}} \\
& \operatorname{LRes}_{2}(s)=V_{2}(s)-\frac{1}{s^{\alpha}}\left(\mathcal{L}\left[\mathcal{L}^{-1}\left[V_{1}(s)\right]^{2}\right]+V_{2}(s)\right) .
\end{aligned}
$$


Accordingly, the $k$-th Laplace residual functions, $L R e s^{k}$, are

$$
\begin{aligned}
& \operatorname{LRes}_{1}^{k}(s)=V_{1}^{k}(s)-\frac{1}{s}-\frac{V_{1}^{k}(s)}{2 s^{\alpha}} \\
& \operatorname{LRes}_{2}^{k}(s)=V_{2}^{k}(s)-\frac{1}{s^{\alpha}}\left(\mathcal{L}\left[\mathcal{L}^{-1}\left[V_{1}^{k}(s)\right]^{2}\right]+V_{2}^{k}(s)\right) .
\end{aligned}
$$

To determine $a_{1}$ and $b_{1}$, we consider

$$
\begin{aligned}
& \operatorname{LRes}_{1}^{1}(s)=V_{1}^{1}(s)-\frac{1}{s}-\frac{V_{1}^{1}(s)}{2 s^{\alpha}} \\
& \operatorname{LRes}_{2}^{1}(s)=V_{2}^{1}(s)-\frac{1}{s^{\alpha}}\left(\mathcal{L}\left[\mathcal{L}^{-1}\left[V_{1}^{1}(s)\right]^{2}\right]+V_{2}^{1}(s)\right) .
\end{aligned}
$$

As $V_{1}^{1}(s)=\frac{1}{s}+\frac{a_{1}}{s^{\alpha+1}}$ and $V_{2}^{1}(s)=\frac{b_{1}}{s^{\alpha+1}}$, we get

$$
\begin{aligned}
\operatorname{LRes}_{1}^{1}(s) & =\frac{a_{1}}{s^{\alpha+1}}-\frac{1}{2 s^{\alpha}}\left(\frac{1}{s}+\frac{a_{1}}{s^{\alpha+1}}\right), \\
\operatorname{LRes}_{2}^{1}(s) & =\frac{b_{1}}{s^{\alpha+1}}-\frac{1}{s^{\alpha}}\left(\mathcal{L}\left[\mathcal{L}^{-1}\left[\frac{1}{s}+\frac{a_{1}}{s^{\alpha+1}}\right]^{2}\right]+\frac{b_{1}}{s^{\alpha+1}}\right) \\
& =\frac{b_{1}}{s^{\alpha+1}}-\frac{1}{s^{\alpha}}\left(\mathcal{L}\left[\left(1+\frac{a_{1} t^{\alpha}}{\Gamma(1+\alpha)}\right)^{2}\right]+\frac{b_{1}}{s^{\alpha+1}}\right) \\
& =\frac{b_{1}}{s^{\alpha+1}}-\frac{1}{s^{\alpha}}\left(\mathcal{L}\left[\left(1+\frac{2 a_{1} t^{\alpha}}{\Gamma(1+\alpha)}+\frac{a_{1}^{2} t^{2 \alpha}}{\Gamma^{2}(1+\alpha)}\right)\right]+\frac{b_{1}}{s^{\alpha+1}}\right) \\
& =\frac{b_{1}}{s^{\alpha+1}}-\frac{1}{s^{\alpha}}\left(\frac{1}{s}+\frac{2 a_{1}}{s^{1+\alpha}}+\frac{a_{1}^{2} \Gamma(1+2 \alpha)}{\Gamma^{2}(1+\alpha) s^{1+2 \alpha}}+\frac{b_{1}}{s^{\alpha+1}}\right) .
\end{aligned}
$$

Multiply (18) by $s^{\alpha+1}$, we obtain that

$$
\begin{aligned}
& s^{\alpha+1} \operatorname{LRes}_{1}^{1}(s)=a_{1}-\frac{1}{2}\left(1+\frac{a_{1}}{s^{\alpha}}\right), \\
& s^{\alpha+1} \operatorname{Les}_{2}^{1}(s)=-\frac{2 a_{1}}{s^{\alpha}}-\frac{a_{1}^{2} \Gamma(2 \alpha+1)}{\Gamma^{2}(\alpha+1) s^{2 \alpha}}+b_{1}\left(1-\frac{1}{s^{\alpha}}\right)-1 .
\end{aligned}
$$

Finally, we solve the following system

$$
\begin{aligned}
& \lim _{s \rightarrow \infty}\left(s^{\alpha+1} \operatorname{Les}_{1}^{1}(s)\right)=0, \\
& \lim _{s \rightarrow \infty}\left(s^{\alpha+1} \operatorname{LRes}_{2}^{1}(s)\right)=0,
\end{aligned}
$$

which gives that

$$
\begin{aligned}
& a_{1}=\frac{1}{2}, \\
& b_{1}=1 .
\end{aligned}
$$

In a similar manner, to find $a_{2}$ and $b_{2}$, we consider

$$
\begin{aligned}
& \operatorname{LRes}_{1}^{2}(s)=V_{1}^{2}(s)-\frac{1}{s}-\frac{V_{1}^{2}(s)}{2 s^{\alpha}} \\
& \operatorname{LRes}_{2}^{2}(s)=V_{2}^{2}(s)-\frac{1}{s^{\alpha}}\left(\mathcal{L}\left[\mathcal{L}^{-1}\left[V_{1}^{2}(s)\right]^{2}\right]+V_{2}^{2}(s)\right) .
\end{aligned}
$$

As $V_{1}^{2}(s)=\frac{1}{s}+\frac{1}{2 s^{\alpha+1}}+\frac{a_{2}}{s^{2 \alpha+1}}$ and $V_{2}^{2}(s)=\frac{1}{s^{\alpha+1}}+\frac{b_{2}}{s^{2 \alpha+1}}$, we obtain

$$
\begin{aligned}
& \operatorname{LRes}_{1}^{2}(s)=\frac{a_{2}}{s^{2 \alpha+1}}-\frac{1}{4 s^{2 \alpha+1}}-\frac{a_{2}}{2 s^{3 \alpha+1}}, \\
& \operatorname{LRes}_{2}^{2}(s)=\frac{1}{s^{\alpha+1}}+\frac{b_{2}}{s^{2 \alpha+1}}-\frac{1}{s^{\alpha}}\left(\mathcal{L}\left[\mathcal{L}^{-1}\left[\frac{1}{s}+\frac{1}{2 s^{\alpha+1}}+\frac{a_{2}}{s^{2 \alpha+1}}\right]^{2}\right]+\frac{1}{s^{\alpha+1}}+\frac{b_{2}}{s^{2 \alpha+1}}\right) .
\end{aligned}
$$


Multiply (23) by $s^{2 \alpha+1}$, and then solve

$$
\begin{aligned}
& \lim _{s \rightarrow \infty}\left(s^{2 \alpha+1} \operatorname{Les}_{1}^{2}(s)\right)=0, \\
& \lim _{s \rightarrow \infty}\left(s^{2 \alpha+1} \operatorname{Les}_{2}^{2}(s)\right)=0,
\end{aligned}
$$

we deduce that

$$
\begin{gathered}
a_{2}=\frac{1}{4}, \\
b_{2}=2 .
\end{gathered}
$$

Hence, the 2nd-approximate LRPS solution of $V_{1}^{2}(s)$ and $V_{2}^{2}(s)$ are

$$
\begin{aligned}
& V_{1}^{2}(s)=\frac{1}{s}+\frac{1}{2 s^{\alpha+1}}+\frac{1}{4 s^{2 \alpha+1}}, \\
& V_{2}^{2}(s)=\frac{1}{s^{\alpha+1}}+\frac{1}{2 s^{2 \alpha+1}} .
\end{aligned}
$$

Proceeding as the above illustrated steps in determining the unknown functions $a_{k}$ and $b_{k}$, one can easily reach the following results:

$$
\begin{aligned}
& a_{3}=\frac{1}{8}, \\
& a_{4}=\frac{1}{16}, \\
& a_{5}=\frac{1}{32},
\end{aligned}
$$

and

$$
\begin{aligned}
& b_{3}=\frac{5}{2}+\frac{\Gamma(1+2 \alpha)}{4 \Gamma^{2}(1+\alpha)}, \\
& b_{4}=\frac{1}{4}\left(11+\frac{\Gamma(1+2 \alpha)}{\Gamma^{2}(1+\alpha)}+\frac{\Gamma(1+3 \alpha)}{\Gamma(1+\alpha) \Gamma(1+2 \alpha)}\right), \\
& b_{5}=\frac{1}{16}\left(46+\frac{4 \Gamma(1+2 \alpha)}{\Gamma^{2}(1+\alpha)}+\frac{\Gamma(1+4 \alpha)}{\Gamma^{2}(1+2 \alpha)}+\frac{1}{\Gamma(1+\alpha)}\left(\frac{4 \Gamma(1+3 \alpha)}{\Gamma(1+2 \alpha)}+\frac{2 \Gamma(1+4 \alpha)}{\Gamma(1+3 \alpha)}\right)\right) .
\end{aligned}
$$

Therefore,

$$
\begin{aligned}
V_{1}(s) & =\frac{1}{s}+\frac{1}{2 s^{\alpha+1}}+\frac{1}{4 s^{2 \alpha+1}}+\frac{1}{8 s^{3 \alpha+1}}+\frac{1}{16 s^{4 \alpha+1}}+\frac{1}{32 s^{5 \alpha+1}}+\ldots, \\
V_{2}(s) & =\frac{1}{s^{\alpha+1}}+\frac{1}{2 s^{2 \alpha+1}}+\frac{1}{s^{3 \alpha+1}}\left(\frac{5}{2}+\frac{\Gamma(1+2 \alpha)}{4 \Gamma^{2}(1+\alpha)}\right)+\frac{1}{s^{4 \alpha+1}}\left(\frac{1}{4}\left(11+\frac{\Gamma(1+2 \alpha)}{\Gamma^{2}(1+\alpha)}+\frac{\Gamma(1+3 \alpha)}{\Gamma(1+\alpha) \Gamma(1+2 \alpha)}\right)\right) \\
& +\frac{1}{s^{5 \alpha+1}}\left(\frac{1}{16}\left(46+\frac{4 \Gamma(1+2 \alpha)}{\Gamma^{2}(1+\alpha)}+\frac{\Gamma(1+4 \alpha)}{\Gamma^{2}(1+2 \alpha)}+\frac{1}{\Gamma(1+\alpha)}\left(\frac{4 \Gamma(1+3 \alpha)}{\Gamma(1+2 \alpha)}+\frac{2 \Gamma(1+4 \alpha)}{\Gamma(1+3 \alpha)}\right)\right)+\ldots .\right.
\end{aligned}
$$

Consequently, the solution of (10)-(11) is

$$
\begin{aligned}
v_{1}(t) & =1+\frac{t^{\alpha}}{2 \Gamma(1+\alpha)}+\frac{t^{2 \alpha}}{4 \Gamma(1+2 \alpha)}+\frac{t^{3 \alpha}}{8 \Gamma(1+3 \alpha)}+\frac{t^{4 \alpha}}{16 \Gamma(1+4 \alpha)}+\frac{t^{5 \alpha}}{32 \Gamma(1+5 \alpha)}+\ldots \\
v_{2}(t) & =\frac{2 t^{2 \alpha}}{\Gamma(1+2 \alpha)}+\frac{10 t^{3 \alpha}}{4 \Gamma(1+3 \alpha)}++\frac{t^{\alpha}}{4 \Gamma^{2}(1+\alpha)}\left(4 \Gamma(1+\alpha)+\frac{t^{2 \alpha} \Gamma(1+2 \alpha)}{\Gamma(1+3 \alpha)}\right) \\
& +\frac{t^{4 \alpha}\left(11 \Gamma^{2}(1+\alpha) \Gamma(1+2 \alpha)+\Gamma^{2}(1+2 \alpha)+\Gamma(1+\alpha) \Gamma(1+3 \alpha)\right)}{4 \Gamma^{2}(1+\alpha) \Gamma(1+2 \alpha) \Gamma(1+4 \alpha)}+\ldots
\end{aligned}
$$

We point out that the exact solutions to the system in Example 1 for the case of $\alpha=1$ are $v_{1}(t)=e^{\frac{t}{2}}$ and $v_{2}(t)=t e^{t}$ $[7,8]$. We consider $\phi_{1}(t)=\sum_{i=0}^{6} a_{i} t^{i \alpha}$ to be the LRPS approximation of $v_{1}(t)$, and $\phi_{2}(t)=\sum_{i=0}^{6} b_{i} t^{i \alpha}$ to be the LRPS approximation of $v_{2}(t)$. In Figure 1, the first sub-figure represents the values of $\phi_{1}(t)$ for different values of the fractional order $\alpha$ and values of $v_{1}(t)$, while the second sub-figure represents the absolute error $\left|v_{1}(t)-\phi_{1}(t)\right|$ when $\alpha=1$. In 
Figure 2, the first sub-figure represents the values of $\phi_{2}(t)$ for different values of the fractional order $\alpha$ and values of $v_{2}(t)$, while the second sub-figure represents the absolute error $\left|v_{2}(t)-\phi_{2}(t)\right|$ when $\alpha=1$. From these plots, we observe that the curves of $\phi_{i}(t): i=1,2$, are mapping continuously and gradually as $\alpha$ varies from 0 to 1 and converges to $v_{i}(t): i=1,2$, when $\alpha=1$. Also, we observe that the approximations $\phi_{i}(t): i=1,2$, are in excellent agreement with $v_{i}(t): i=1,2$, when $\alpha=1$.

On the other side, as shown in Table 1, we provide numerical investigations on the accuracy of LRPS applied to Example 1. While as, in Table 2, we present the impact of the fractional order $\alpha$ acting on the values of the unknowns field functions $v_{i}(t): i=1,2$.
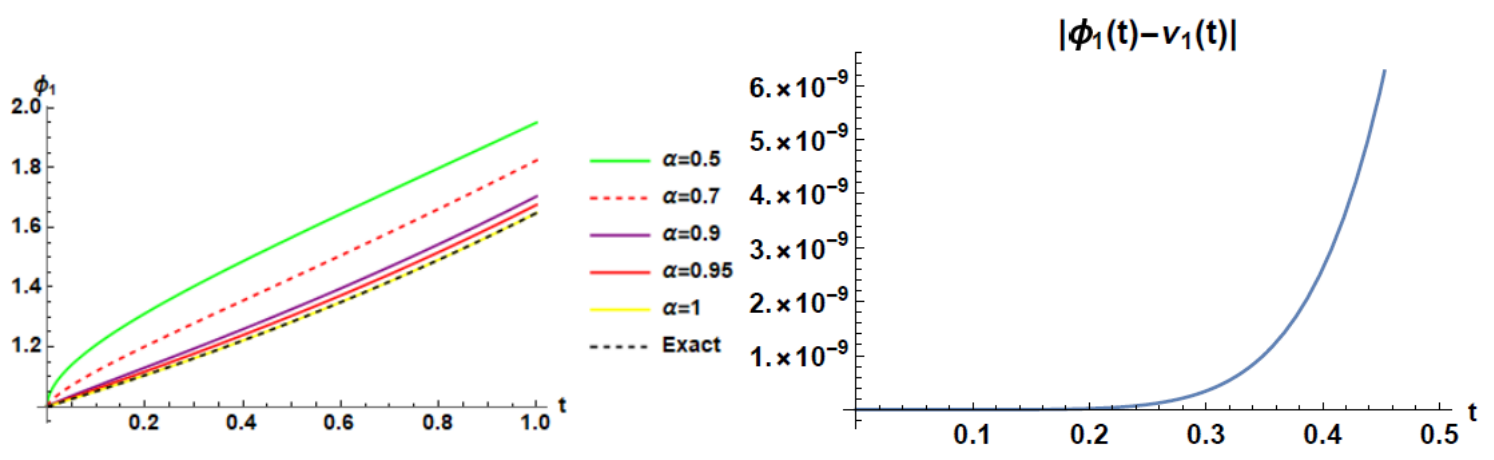

Figure 1: Exact, profile approximate solutions and absolute error regarding $v_{1}$ of Example 1.

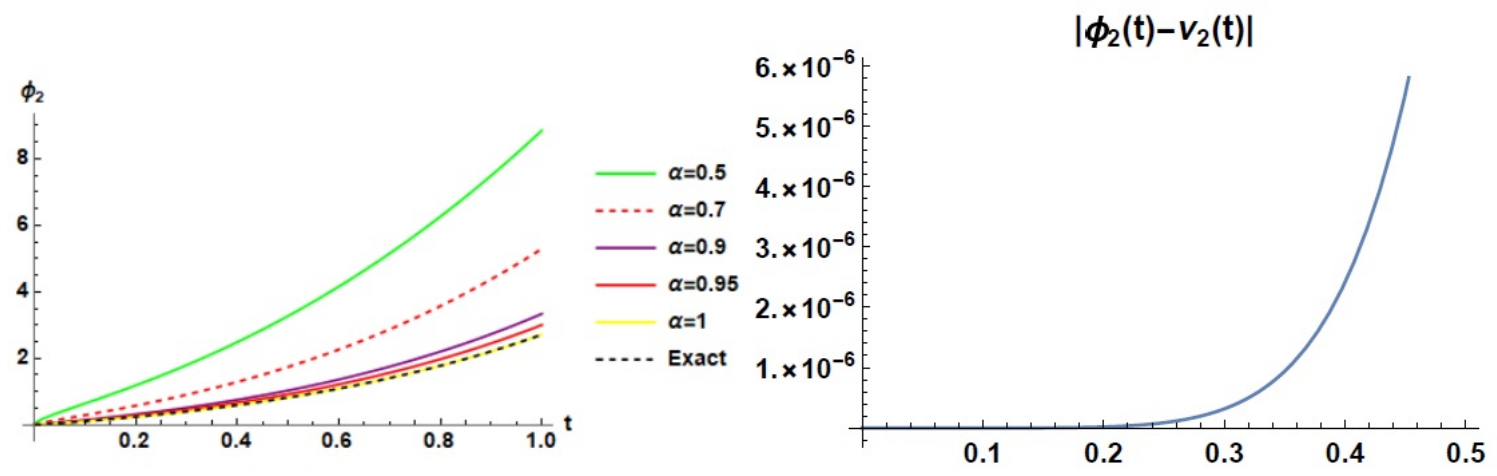

Figure 2: Exact, profile approximate solutions and absolute error regarding $v_{2}$ of Example 1.

Table 1: Numerical values of $\phi_{1}(t)$ and $\phi_{2}(t)$ for $\alpha=0.5,0.7,0.9$ to Example 1.

\begin{tabular}{c|c|c|c|c|c|c}
\hline \multirow{2}{*}{$t$} & \multicolumn{2}{|c|}{$\alpha=0.5$} & \multicolumn{2}{c|}{$\alpha=0.7$} & \multicolumn{2}{c}{$\alpha=0.9$} \\
\cline { 2 - 7 } & $v_{1}(t)$ & $v_{2}(t)$ & $v_{1}(t)$ & $v_{2}(t)$ & $v_{1}(t)$ & $v_{2}(t)$ \\
\hline 0.2 & 1.312141902 & 1.184140706 & 1.201599157 & 0.580240824 & 1.130764596 & 0.320222598 \\
\hline 0.4 & 1.486564677 & 2.444376604 & 1.354985531 & 1.290827616 & 1.259270690 & 0.756805142 \\
\hline 0.6 & 1.6445934256 & 4.006862318 & 1.506375497 & 2.249749087 & 1.396080779 & 1.365217628 \\
\hline 0.8 & 1.7972927210 & 5.900827955 & 1.661915587 & 3.516856974 & 1.543797927 & 2.198213783 \\
\hline
\end{tabular}


Table 2: Absolute errors: $\left|\phi_{1}(t)-v_{1}(t)\right|$ and $\left|\phi_{2}(t)-v_{2}(t)\right|$ to Example 1.

\begin{tabular}{c|c|c}
\hline$t$ & $\left|\phi_{1}(t)-v_{1}(t)\right|$ & $\left|\phi_{2}(t)-v_{2}(t)\right|$ \\
\hline 0.2 & $1.408981153 \times 10^{-9}$ & $5.516320339 \times 10^{-7}$ \\
\hline 0.4 & $9.149350321 \times 10^{-8}$ & $3.65457231 \times 10^{-5}$ \\
\hline 0.6 & $1.057576003 \times 10^{-6}$ & $4.31280234 \times 10^{-4}$ \\
\hline 0.8 & $6.030974603 \times 10^{-6}$ & $2.51274279 \times 10^{-3}$ \\
\hline
\end{tabular}

\subsection{Example 2}

Let us consider the following $3 \times 3$ nonlinear autonomous system $[9,10]$

$$
\left\{\begin{array}{l}
D^{\alpha} v_{1}(t)=v_{1}(t), \\
D^{\alpha} v_{2}(t)=2\left(v_{1}(t)\right)^{2}, \\
D^{\alpha} v_{3}(t)=3 v_{1}(t) v_{2}(t) ;
\end{array}\right.
$$

subject to

$$
\begin{aligned}
& v_{1}(0)=1, \\
& v_{2}(0)=1, \\
& v_{3}(0)=0 .
\end{aligned}
$$

Apply the Laplace transform to (31)-(32), we get

$$
\begin{aligned}
& V_{1}(s)=\frac{1}{s}+\frac{V_{1}(s)}{s^{\alpha}} \\
& V_{2}(s)=\frac{1}{s}+\frac{2}{s^{\alpha}}\left(\mathcal{L}\left[\mathcal{L}^{-1}\left[V_{1}(s)\right]^{2}\right]\right), \\
& V_{3}(s)=\frac{3 \mathcal{L}\left[\mathcal{L}^{-1}\left[V_{1}(s)\right] \mathcal{L}^{-1}\left[V_{2}(s)\right]\right]}{s^{\alpha}},
\end{aligned}
$$

Assume that $V_{1}(s), V_{2}(s)$ and $V_{3}(s)$ have fractional power series as

$$
\begin{aligned}
& V_{1}(s)=\sum_{n=0}^{\infty} \frac{a_{n}}{s^{n \alpha+1}}, \\
& V_{2}(s)=\sum_{n=0}^{\infty} \frac{b_{n}}{s^{n \alpha+1}}, \\
& V_{3}(s)=\sum_{n=0}^{\infty} \frac{c_{n}}{s^{n \alpha+1}},
\end{aligned}
$$

with the $k$-th truncated series

$$
\begin{aligned}
& V_{1}^{k}(s)=\sum_{n=0}^{k} \frac{a_{n}}{s^{n \alpha+1}}=\frac{1}{s}+\sum_{n=1}^{k} \frac{a_{n}}{s^{n \alpha+1}}, \\
& V_{2}^{k}(s)=\sum_{n=0}^{k} \frac{b_{n}}{s^{n \alpha+1}}=\frac{1}{s}+\sum_{n=1}^{k} \frac{b_{n}}{s^{n \alpha+1}}, \\
& V_{3}^{k}(s)=\sum_{n=0}^{k} \frac{c_{n}}{s^{n \alpha+1}}=\sum_{n=1}^{k} \frac{c_{n}}{s^{n \alpha+1}}, \text { respectively, }
\end{aligned}
$$

whose the Laplace residual functions for $V_{1}^{k}(s), V_{2}^{k}(s)$ and $V_{3}^{k}(s)$ are

$$
\begin{aligned}
& \operatorname{LRes}_{1}(s)=V_{1}(s)-\frac{1}{s}-\frac{V_{1}(s)}{s^{\alpha}}, \\
& \operatorname{LRes}_{2}(s)=V_{2}(s)-\frac{1}{s}-\frac{1}{s^{\alpha}}\left(\mathcal{L}\left[\mathcal{L}^{-1}\left[V_{1}(s)\right]^{2}\right]\right), \\
& \operatorname{LRes}_{3}(s)=V_{3}(s)-\frac{3 \mathcal{L}\left[\mathcal{L}^{-1}\left[V_{1}(s)\right] \mathcal{L}^{-1}\left[V_{2}(s)\right]\right]}{s^{\alpha}} .
\end{aligned}
$$


Accordingly, the $k$-th Laplace residual functions, $\operatorname{LRes}^{k}$, are

$$
\begin{aligned}
& \operatorname{LRes}_{1}^{k}(s)=V_{1}^{k}(s)-\frac{1}{s}-\frac{V_{1}^{k}(s)}{s^{\alpha}}, \\
& \operatorname{LRes}_{2}^{k}(s)=V_{2}^{k}(s)-\frac{1}{s}-\frac{1}{s^{\alpha}}\left(\mathcal{L}\left[\mathcal{L}^{-1}\left[V_{1}^{k}(s)\right]^{2}\right]\right), \\
& \operatorname{LRes}_{3}^{k}(s)=V_{3}^{k}(s)-\frac{3 \mathcal{L}\left[\mathcal{L}^{-1}\left[V_{1}^{k}(s)\right] \mathcal{L}^{-1}\left[V_{2}^{k}(s)\right]\right]}{s^{\alpha}},
\end{aligned}
$$

To determine $a_{1}, b_{1}$ and $c_{1}$, we substitute (35) in (37) with $k=1$ to get

$$
\begin{aligned}
& \operatorname{LRes}_{1}^{1}(s)=\frac{a_{1}}{s^{\alpha+1}}-\frac{1}{s^{\alpha}}\left(\frac{1}{s}+\frac{a_{1}}{s^{\alpha+1}}\right), \\
& \operatorname{LRes}_{2}^{1}(s)=\frac{b_{1}}{s^{\alpha+1}}-\frac{1}{s^{\alpha}}\left(\mathcal{L}\left[\mathcal{L}^{-1}\left[\frac{1}{s}+\frac{a_{1}}{s^{\alpha+1}}\right]^{2}\right]\right), \\
& \operatorname{LRes}_{3}^{1}(s)=\frac{c_{1}}{s^{\alpha+1}}-\frac{3}{s^{\alpha}}\left(\mathcal{L}\left[\mathcal{L}^{-1}\left[\frac{1}{s}+\frac{a_{1}}{s^{\alpha+1}}\right] \mathcal{L}^{-1}\left[\frac{1}{s}+\frac{b_{1}}{s^{\alpha+1}}\right]\right]\right),
\end{aligned}
$$

Multiply (38) by $s^{\alpha+1}$,

$$
\begin{aligned}
& s^{\alpha+1} \operatorname{LRes}_{1}^{1}(s)=a_{1}-\left(1+\frac{a_{1}}{s^{\alpha}}\right), \\
& s^{\alpha+1} \operatorname{LRes}_{2}^{1}(s)=b_{1}-s\left(\mathcal{L}\left[\mathcal{L}^{-1}\left[\frac{1}{s}+\frac{a_{1}}{s^{\alpha+1}}\right]^{2}\right]\right), \\
& s^{\alpha+1} \operatorname{LRes}_{3}^{1}(s)=c_{1}-3 s\left(\mathcal{L}\left[\mathcal{L}^{-1}\left[\frac{1}{s}+\frac{a_{1}}{s^{\alpha+1}}\right] \mathcal{L}^{-1}\left[\frac{1}{s}+\frac{b_{1}}{s^{\alpha+1}}\right]\right]\right) .
\end{aligned}
$$

It is clear that when we solve $\lim _{s \rightarrow \infty}\left(s^{\alpha+1} \operatorname{LRes}_{m}^{1}(s)\right)=0, m=1,2,3$, we directly obtain

$$
\begin{aligned}
& a_{1}=1, \\
& b_{1}=2, \\
& c_{1}=3 .
\end{aligned}
$$

Similarly, when we substitute (35) in (37) with $k=2$ and then use the fact that $\lim _{s \rightarrow \infty}\left(s^{\alpha+1} \operatorname{LRes}_{m}^{2}(s)\right)=0, m=1,2,3$, we reach

$$
\begin{aligned}
& a_{2}=1, \\
& b_{2}=4, \\
& c_{2}=9 .
\end{aligned}
$$

Hence, the $2^{\text {nd }}$-approximate LRPS solution of $V_{1}^{2}(s), V_{2}^{2}(s)$ and $V_{3}^{2}(s)$ are

$$
\begin{aligned}
& V_{1}^{2}(s)=\frac{1}{s}+\frac{1}{s^{\alpha+1}}+\frac{1}{s^{2 \alpha+1}}, \\
& V_{2}^{2}(s)=\frac{1}{s}+\frac{2}{s^{\alpha+1}}+\frac{4}{s^{2 \alpha+1}}, \\
& V_{3}^{2}(s)=\frac{3}{s^{\alpha+1}}+\frac{9}{s^{2 \alpha+1}} .
\end{aligned}
$$

Proceeding as the above illustrated steps in determining the unknown functions $a_{k}, b_{k}$ and $c_{k}$, one can easily verify the following results:

$$
\begin{aligned}
& a_{3}=1, \\
& a_{4}=1, \\
& a_{5}=1,
\end{aligned}
$$




$$
\begin{gathered}
b_{3}=4+\frac{2 \Gamma(1+2 \alpha)}{\Gamma^{2}(1+\alpha)}, \\
b_{4}=4+\frac{4 \Gamma(1+3 \alpha)}{\Gamma(1+\alpha) \Gamma(1+2 \alpha)}, \\
b_{5}=4+2\left(\frac{1}{\Gamma^{2}(1+2 \alpha)}+\frac{2}{\Gamma(1+\alpha) \Gamma(1+3 \alpha)}\right) \Gamma(1+4 \alpha), \\
c_{3}=15+\frac{6 \Gamma(1+2 \alpha)}{\Gamma^{2}(1+\alpha)} \\
c_{4}=15+\frac{6\left(\Gamma^{2}(1+2 \alpha)+3 \Gamma(1+\alpha) \Gamma(1+3 \alpha)\right)}{\Gamma^{2}(1+\alpha) \Gamma(1+2 \alpha)}, \\
c_{5}=3\left(5+\frac{4 \Gamma(1+4 \alpha)}{\Gamma^{2}(1+2 \alpha)}+\frac{2 \Gamma(1+2 \alpha) \Gamma(1+4 \alpha)}{\Gamma^{3}(1+\alpha) \Gamma(1+3 \alpha)}+\frac{1}{\Gamma(1+\alpha)}\left(\frac{4 \Gamma(1+3 \alpha)}{\Gamma(1+2 \alpha)}+\frac{6 \Gamma(1+4 \alpha)}{\Gamma(1+3 \alpha)}\right)\right) .
\end{gathered}
$$

Therefore,

$$
\begin{aligned}
V_{1}(s) & =\frac{1}{s}+\frac{1}{s^{\alpha+1}}+\frac{1}{s^{2 \alpha+1}}+\frac{1}{s^{3 \alpha+1}}+\frac{1}{s^{4 \alpha+1}}+\frac{1}{s^{5 \alpha+1}}+\ldots, \\
V_{2}(s) & =\frac{1}{s}+\frac{2}{s^{\alpha+1}}+\frac{4}{s^{2 \alpha+1}}+\frac{1}{s^{3 \alpha+1}}\left(4+\frac{2 \Gamma(1+2 \alpha)}{\Gamma(1+\alpha)^{2}}\right)+\frac{1}{s^{4 \alpha+1}}\left(4+\frac{4 \Gamma(1+3 \alpha)}{\Gamma(1+\alpha) \Gamma(1+2 \alpha)}\right) \\
& +\frac{1}{s^{5 \alpha+1}}\left(4+2\left(\frac{1}{\Gamma(1+2 \alpha)^{2}}+\frac{2}{\Gamma(1+\alpha) \Gamma(1+3 \alpha)}\right) \Gamma(1+4 \alpha)\right)+\ldots, \\
V_{3}(s) & =\frac{3}{s^{\alpha+1}}+\frac{9}{s^{2 \alpha+1}} \\
& +\frac{1}{s^{3 \alpha+1}}\left(15+\frac{6 \Gamma(1+2 \alpha)}{\Gamma(1+\alpha)^{2}}\right)+\frac{1}{s^{4 \alpha+1}}\left(15+\frac{6\left(\Gamma(1+2 \alpha)^{2}+3 \Gamma(1+\alpha) \Gamma(1+3 \alpha)\right)}{\Gamma(1+\alpha)^{2} \Gamma(1+2 \alpha)}\right) \\
& +\frac{3\left(5+\frac{4 \Gamma(1+4 \alpha)}{\Gamma(1+2 \alpha)^{2}}+\frac{2 \Gamma(1+2 \alpha) \Gamma(1+4 \alpha)}{\Gamma(1+\alpha)^{3} \Gamma(1+3 \alpha)}+\frac{1}{\Gamma(1+\alpha)}\left(\frac{4 \Gamma(1+3 \alpha)}{\Gamma(1+2 \alpha)}+\frac{6 \Gamma(1+4 \alpha)}{\Gamma(1+3 \alpha)}\right)\right)}{s^{5 \alpha+1}}+\ldots .
\end{aligned}
$$

Consequently, the solution of (31)-(32) is

$$
\begin{aligned}
v_{1}(t) & =1+\frac{t^{\alpha}}{\Gamma(1+\alpha)}+\frac{t^{2 \alpha}}{\Gamma(1+2 \alpha)}+\frac{t^{3 \alpha}}{\Gamma(1+3 \alpha)}+\frac{t^{4 \alpha}}{\Gamma(1+4 \alpha)}+\frac{t^{5 \alpha}}{\Gamma(1+5 \alpha)}+\ldots, \\
v_{2}(t) & =1+\frac{4 t^{2 \alpha}}{\Gamma(1+2 \alpha)}+\frac{4 t^{3 \alpha}}{\Gamma(1+3 \alpha)}+\frac{2 t^{3 \alpha} \Gamma(1+2 \alpha)}{\Gamma(1+\alpha)^{2} \Gamma(1+3 \alpha)}+\frac{2 t^{\alpha}+\frac{4 t^{4 \alpha} \Gamma(1+3 \alpha)}{\Gamma(1+2 \alpha) \Gamma(1+4 \alpha)}}{\Gamma(1+\alpha)} \\
& +\frac{4 t^{4 \alpha}}{\Gamma(1+4 \alpha)}+\frac{1}{\Gamma(1+5 \alpha)}\left(t^{5 \alpha}\left(4+2\left(\frac{1}{\Gamma(1+2 \alpha)^{2}}+\frac{2}{\Gamma(1+\alpha) \Gamma(1+3 \alpha)}\right) \Gamma(1+4 \alpha)\right)\right)+\ldots, \\
v_{3}(t) & =t^{\alpha}\left(3 \left(t^{\alpha}\left(\frac{3}{\Gamma(1+2 \alpha)}+5 t^{\alpha}\left(\frac{1}{\Gamma(1+3 \alpha)}+\frac{t^{\alpha}}{\Gamma(1+4 \alpha)}\right)\right)\right.\right. \\
& \left.+\frac{1}{\Gamma(1+\alpha)^{2}}\left(2 t^{2 \alpha} \Gamma(1+2 \alpha)\left(\frac{1}{\Gamma(1+3 \alpha)}+\frac{1}{\Gamma(1+4 \alpha)}\right)\right)+\frac{\left.\left.1+\frac{6 t^{3 \alpha} \Gamma(1+3 \alpha)}{\Gamma(1+2 \alpha) \Gamma(1+4 \alpha)}\right)\right)+\ldots .}{\Gamma(1+\alpha)}\right)
\end{aligned}
$$

It is worth mentioning that the exact solutions to the system in Example 2 for the case $\alpha=1$ are $v_{1}(t)=e^{t}, v_{2}(t)=e^{2 t}$ and $v_{3}(t)=e^{3 t}-1[9,10]$. We consider $\psi_{1}(t)=\sum_{i=0}^{6} a_{i} t^{i \alpha}$ to be the LRPS approximation of $v_{1}(t), \psi_{2}(t)=\sum_{i=0}^{6} b_{i} t^{i \alpha}$ to be the LRPS approximation of $v_{2}(t)$ and $\psi_{3}(t)=\sum_{i=0}^{6} c_{i} t^{i \alpha}$ to be the LRPS approximation of $v_{3}(t)$. In Figure 3 , the first subfigure represents the values of $\psi_{1}(t)$ for different values of the fractional order $\alpha$ and values of $v_{1}(t)$, while the second sub-figure represents the absolute error $\left|v_{1}(t)-\psi_{1}(t)\right|$ when $\alpha=1$. In Figure 4, the first sub-figure represents the values of $\psi_{2}(t)$ for different values of the fractional order $\alpha$ and values of $v_{2}(t)$, while the second sub-figure represents the absolute error $\left|v_{2}(t)-\psi_{2}(t)\right|$ when $\alpha=1$. In Figure 5, the first sub-figure represents the values of $\psi_{3}(t)$ for different values of the fractional order $\alpha$ and values of $v_{3}(t)$, while the second sub-figure represents the absolute error $\left|v_{3}(t)-\psi_{3}(t)\right|$ when $\alpha=1$. For this $3 \times 3$ system, one can observe the same findings depicted for Example 1. Finally, as shown in Table 3, we provide numerical investigations on the accuracy of LRPS applied to Example 2. 

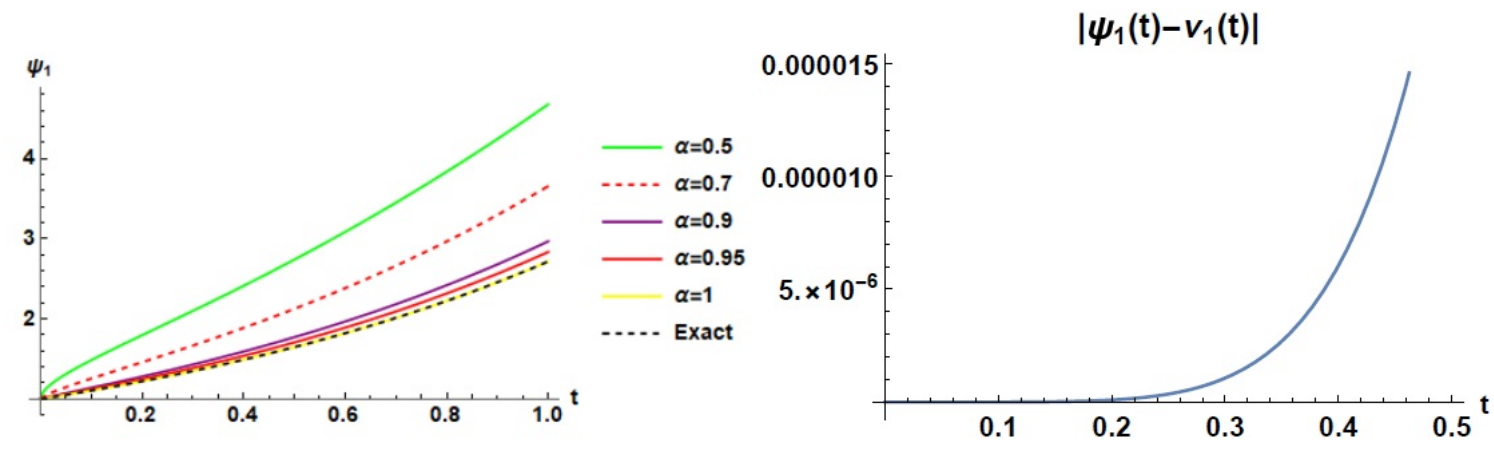

Figure 3: Exact, profile approximate solutions and absolute error regarding $v_{1}$ of Example 2.

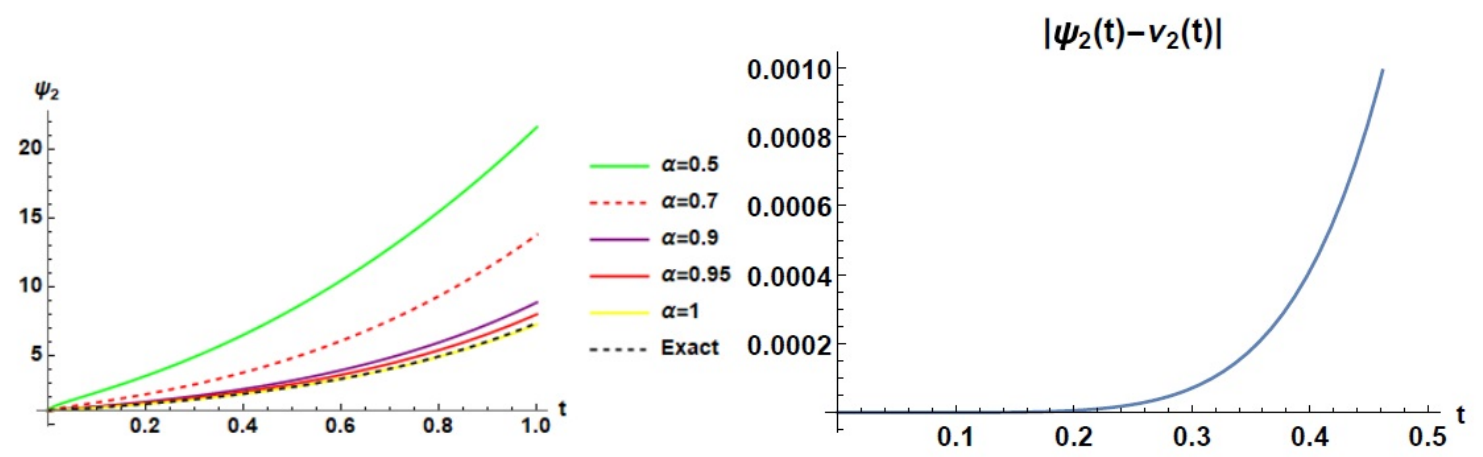

Figure 4: Exact, profile approximate solutions and absolute error regarding $v_{2}$ of Example 2.

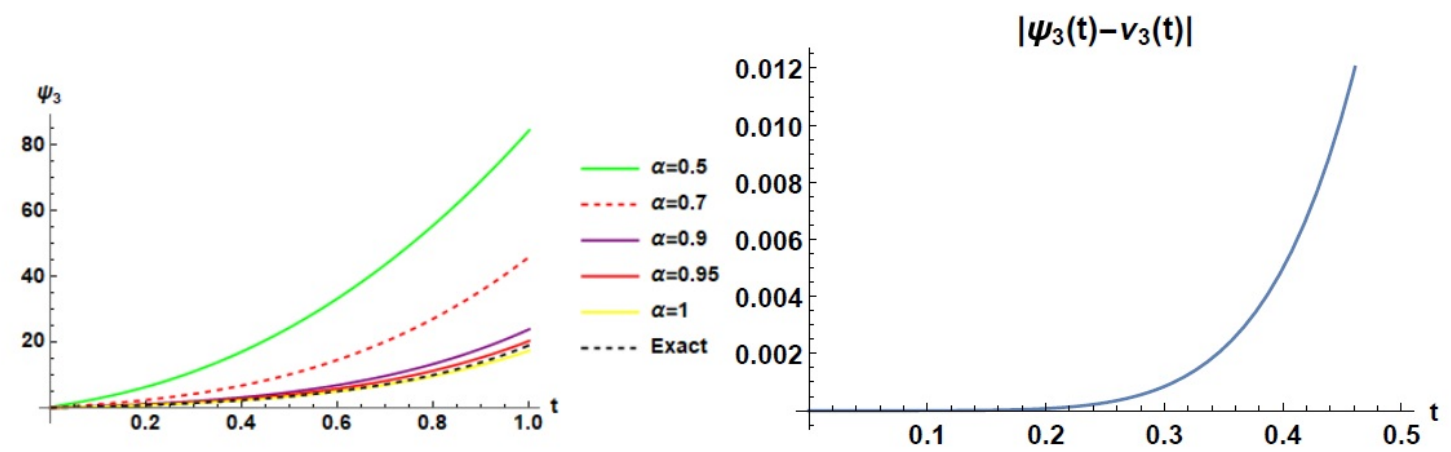

Figure 5: Exact, profile approximate solutions and absolute error regarding $v_{3}$ of Example 2.

Table 3: Absolute errors: $\left|\psi_{1}(t)-v_{1}(t)\right|,\left|\psi_{2}(t)-v_{2}(t)\right|$ and $\left|\psi_{3}(t)-v_{3}(t)\right|$ to Example 2 .

\begin{tabular}{c|c|c|c}
\hline$t$ & $\left|\psi_{1}(t)-v_{1}(t)\right|$ & $\left|\psi_{2}(t)-v_{2}(t)\right|$ & $\left|\psi_{3}(t)-v_{3}(t)\right|$ \\
\hline 0.2 & $9.149350315 \times 10^{-8}$ & $6.030974603 \times 10^{-6}$ & $7.080039050 \times 10^{-5}$ \\
\hline 0.4 & $6.030974603 \times 10^{-6}$ & $4.102618258 \times 10^{-4}$ & $4.980922736 \times 10^{-3}$ \\
\hline 0.6 & $7.080039050 \times 10^{-5}$ & $4.980922736 \times 10^{-3}$ & $6.278346441 \times 10^{-2}$ \\
\hline 0.8 & $4.102618258 \times 10^{-4}$ & $2.991775772 \times 10^{-2}$ & $3.932243806 \times 10^{-1}$ \\
\hline
\end{tabular}




\section{Conclusion}

A combination of two schemes; the Laplace transform and the residual power series, is adapted to solve nonlinear Caputo-fractional autonomous dynamical systems. The methodology, reliability and the accuracy of the new technique are introduced by solving $2 \times 2$ and $3 \times 3$ systems. The role of the fractional derivative is investigated by using graphical analysis. Finally, the advantage of the current method was depicted as converting the whole fractional problem into pure algebraic computational scheme which can be executed using any available computational softwares.

As a future work, the authors plan to extend the use of LRPS to solve multi-dimensional various fractional problems arising in Engineering and Science.

Funding information: The authors state no funding involved.

Author contributions: All authors have accepted responsibility for the entire content of this manuscript and approved its submission.

Conflict of interest: The authors state no conflict of interest.

\section{References}

[1] Almeida R, Guzowska M, Odzijewicz T. A remark on local fractional calculus and ordinary derivatives. Open Math. 2016;14:1122-1124.

[2] Razzaghi M, Ordokhani Y. Solution of nonlinear Volterra- Hammerstein integral equations via rationalized Haar functions. Mathe. Probl. Eng. 2001;7(2):205-219.

[3] Owolabi KM, Hammouch Z. Spatiotemporal patterns in the Belousov-Zhabotinskii reaction systems with AtanganaBaleanu fractional order derivative. Phys. A: Stat. Mech. Appl. 2019;523:1072-1090.

[4] Keshavarz E, Ordokhani Y, Razzaghi M. A numerical solution for fractional optimal control problems via Bernoulli polynomials. J. Vib. Control. 2016;22(18):3889-3903.

[5] Ejlali N, Hosseini SM, Yousefi SA. B-spline spectral method for constrained fractional optimal control problems. Mathe. Methods Appl. Sci. 2018;41(14):5466-5480.

[6] Ganji RM, Jafari H. A new approach for solving nonlinear Volterra integro-differential equations with Mittag-Leffler kernel. Proc. Inst. Mathe. Mech. 2020;46(1):144-158.

[7] Jafari H, Firoozjaee MA, Johnston SJ. An effective approach to solve a system fractional differential equations. Alexandria Engineering Journal. 2020;59(5):3213-3219.
[8] Dixit S, Singh 0, Kumar S. An analytic algorithm for solving system of fractional differential equations. Journal of Modern Methods in Numerical Mathematics. 2010;1(1):12-26.

[9] Zurigat M, Momani S, Odibat Z, Alawneh A. The homotopy analysis method for handling systems of fractional differential equations. Applied Mathematical Modelling. 2010;34(1):24-35.

[10] Wu XY, Xia JL. Two low accuracy methods for stiff systems. Applied mathematics and computation. 2001;123(2):141-153.

[11] Eriqat T, El-Ajou A, Oqielat MN, Al-Zhour Z, Momani S. A new attractive analytic approach for solutions of linear and nonlinear Neutral fractional Pantograph equations. Chaos, Solitons \& Fractals. 2020;138:109957.

[12] Alquran M, Ali M, Alsukhour M, Jaradat I. Promoted residual power series technique with Laplace transform to solve some time-fractional problems arising in physics. Results in Physics. 2020;19:103667.

[13] Ali M, Alquran M, Jaradat I. Asymptotic-sequentially solution style for the generalized Caputo time-fractional NewellWhitehead-Segel system. Advances in Difference Equations. 2019;2019:70.

[14] Dehghan M, Manafian J, Saadatmandi A. Solving nonlinear fractional partial differential equations using the homotopy analysis method. Numerical Methods for Partial Differential Equations. 2010;26:448-479.

[15] Ganjiani M. Solution of nonlinear fractional differential equations using Homotopy analysis method. Appl. Math. Model. 2010;34:1634-1641.

[16] Alquran M, Jaradat I. Delay-asymptotic solutions for the timefractional delay-type wave equation. Physica A: Statistical Mechanics and its Applications. 2019;527:121275.

[17] Alquran M. Analytical solution of time-fractional twocomponent evolutionary system of order 2 by residual power series method. Journal of Applied Analysis and Computation. 2015;5(4):589-599.

[18] He JH. Homotopy perturbation method: a new nonlinear analytical technique. Appl. Math. Comput. 2003;135:73-79.

[19] Ali M, Alquran M, Jaradat I, Abu Afouna N, Baleanu D. Dynamics of integer-fractional time-derivative for the new two-mode Kuramoto-Sivashinsky model. Rom. Rep. Phys. 2020;72(1):103.

[20] Alquran M, Jaradat I, Momani S, Baleanu D. Chaotic and solitonic solutions for a new time-fractional two-mode Kortewegde Vries equation. Rom. Rep. Phys. 2020;72(3):117.

[21] Abu Irwaq I, Alquran M, Jaradat I, Noorani MSM, Momani S, Baleanu D. Numerical investigations on the physical dynamics of the coupled fractional Boussinesq-Burgers system. Romanian Journal of Physics. 2020;65(5-6):111.

[22] Jaradat I, Al-Dolat M, Al-Zoubi K, Alquran M. Theory and applications of a more general form for fractional power series expansion. Chaos Solitons Fract. 2018;108:107-110.

[23] Jaradat I, Alquran M, Al-Dolat M. Analytic solution of homogeneous time-invariant fractional IVP. Adv. Differ. Equ. 2018;2018:143.

[24] Abu Irwaq I, Alquran M, Ali M, Jaradat I, Noorani MSM. Attractive new fractional-integer power series method for solving singulary perturbed differential equations involving mixed fractional and integer derivatives. Results in Physic. 2021;20:103780.

[25] Jaradat I, Alquran M, Abdel-Muhsen R. An analytical framework of 2D diffusion, wave-like, telegraph, and Burgers' models 
with twofold Caputo derivatives ordering. Nonlinear Dynamics. 2018;93(4):1911-1922.

[26] Alquran M, Al-Khaled K, Sivasundaram S, Jaradat HM. Mathematical and numerical study of existence of bifurcations of the generalized fractional Burgers-Huxley equation. Nonlinear Studies. 2017;24(1):235-244.

[27] El-Ajou A, Abu Arqub O, Al-Smadi M. A general form of the generalized Taylor's formula with some applications. Appl. Math. Comput. 2015;256:851-859.

[28] Komashynska I, Al-Smadi M, Abu Arqub O, Momani S. An efficient analytical method for solving singular initial value problems of nonlinear systems. Appl. Math. Inf. Sci. 2016;10(2):647-656.

[29] Alquran M, Yousef F, Alquran F, Sulaiman TA, Yusuf A. Dualwave solutions for the quadratic-cubic conformable-Caputo time-fractional Klein-Fock-Gordon equation. Mathematics and Computers in Simulation. 2021;185:62-76. 